\title{
El órgano Legislativo: ¿un verdadero espacio de representación nacional?
}

\author{
Editado por/Edited by: Johanna Andrango/ Eduardo Castro \\ Recibido/Received: 9/11/2014. Aceptado/Accepted: 16/05/2015 \\ Publicado en línea/Published on Web: 16/10/2015
}

\author{
María Bernarda Carpio \\ Universidad San Francisco de Quito, Colegio de Jurisprudencia JUR \\ Diego de Robles y Vía Interoceánica, Quito, Ecuador
}

Correo Electrónico: mbernarda.carpio@gmail.com

\section{Resumen}

La democracia se presenta como el régimen de representación popular por excelencia, constituyéndose de este modo el órgano Legislativo como un mecanismo idóneo para plasmar la voluntad popular; de suerte que la Asamblea Nacional logre perfilarse como un espacio de debate y discusión, en el cual diversos intereses sociales se encuentren plasmados. Sin embargo, para alcanzar dicha representación total, resulta indispensable que en este órgano se vean reflejados los intereses de los distintos grupos sociales, ya sean estos mayoritarios o no. No obstante, ante la presencia de una mayoría calificada en el Legislativo, la posibilidad de debate se ve reducida y aquello genera la imposibilidad de que las minorías parlamentarias sean efectivamente representadas. Es indudable que en la realidad nacional el asunto de la efectiva representatividad en el Legislativo se configura como un inconveniente de suprema importancia. Por lo tanto, conviene cuestionarnos, ¿hasta qué punto el poder Legislativo cumple su primordial función de órgano de representación popular ante la existencia de una mayoría absoluta en la Asamblea Nacional? Para responder a aquella interrogante, se analizará en primer lugar la naturaleza de la función legislativa dentro de un sistema democrático; posteriormente se examinará si es plausible atribuir a la instauración del método D’Hondt responsabilidad por los resultados obtenidos en la atribución de escaños en la última elección legislativa nacional, para finalmente estudiar las consecuencias de la presencia de una mayoría absoluta dentro de la representatividad del poder Legislativo.

Palabras clave: Democracia, voluntad popular, representación proporcional, poder Legislativo, división de poderes, método D’Hondt, mayoría absoluta.

\begin{abstract}
Democracy comes as the ideal regime of popular representation, thus the legislature is constituted as a suitable mechanism to reflect the popular will. Therefore, since this is a multi-person power, it allows the representation of minority power groups through debate and discussion, in which various social interests are reflected. However, in the presence of an absolute majority in the Assembly, the possibility of discussion is reduced and generates the inability of parliamentary minorities to be effectively represented. In this sense, it is important to question until what extent the legislature fulfills its primary function of popular representation, when there exists an absolute majority in the Assembly. To answer this question, first it will be discussed the nature of the legislative function within a democratic system; then the existence of a link between the system of proportional representation and the results obtained; and finally it will be study the consequences of the presence of an absolute majority in the representativeness of the Assembly.
\end{abstract}

Keywords: Democracy, popular will, proportional representation, legislature, separation of powers, D'Hondt method, absolute majority. 
Para que un sistema sea democrático, se exige al menos que la mayoría no tenga el poder de suprimir la posibilidad de que las minorías se conviertan en mayorías". Luigi Ferrajoli.

\section{La representatividad como fundamento del órgano Legislativo}

De la etimología griega de la palabra democracia se percibe de manera inmediata que aquella implica la inclusión del pueblo en el poder, pues demos significa pueblo y kratos poder. Sin embargo, para que dicha forma de gobierno sea efectiva es indispensable la adopción de un mecanismo que permita canalizar la voluntad del pueblo: así, la democracia representativa se perfila como el mecanismo eficiente para alcanzarlo.

Dicho proceso se da mediante elecciones populares, en las cuales los electores manifiestan su voluntad de ser representados por determinados candidatos, que se presentan como aquellos que reflejarán de manera idónea y cabal sus intereses. En este sentido, el poder Legislativo halla razón de ser siempre y cuando refleje la voluntad popular, ya que es fundamental que dentro del mismo se dé un proceso de diálogo y debate entre las distintas ideas e intereses. Así se logra un consenso de los mismos y que, sin importar la decisión tomada, ésta respete los derechos de ambas partes.

\section{I.1. Teoría de la separación de poderes como mecanismo garante de la democracia}

En un Estado de Derecho "todos los poderes, incluido el Legislativo, están vinculados al respecto de principios sustanciales, establecidos por las normas constitucionales, como la división de poderes y los derechos fundamentales" (Ferrajoli, s.f., p. 31). En este sentido, los derechos y garantías de los ciudadanos constituyen el eje de este modelo de gobierno. Ahora bien, para lograr la existencia de una verídica protección de los mismos resulta indispensable la existencia de un sistema de separación de poderes puesto que:

El sentido histórico e ideológico del principio de separación de poderes es (...) evitar la concentración de poder en manos, sobre todo, del titular del Ejecutivo, a fin de lograr el respeto a los derechos y libertades de los ciudadanos que, representados democráticamente, constituyen ahora el poder Legislativo (Díaz, 1975, p. 33).

Lo mencionado halla razón en que precisamente en los sistemas autoritarios el poder recaía únicamente sobre el soberano, quien no tenía más límites que los que el mismo se fijaba pues "en el Estado absoluto, quien ostenta el poder es la autoridad, que somete al sistema jurídico a su voluntad" (Ávila, 2008, p. 29). Bajo estas circunstancias se imposibilitaba la existencia de respeto alguno hacia los derechos de los ciudadanos, ya que los intereses del soberano primaban por sobre los de los demás.

Por tanto, el principio de separación de poderes se convierte en una garantía fundamental para garantizar el apego a la ley y el correlativo acatamiento a los derechos fundamentales. Siendo así, el pueblo- y el respeto a sus derechos y libertades- constituyen la fuente del límite al ejercicio del poder; en vista de que "los derechos de las personas son, a la vez, límites del poder y vínculos. Límites porque ningún poder los puede violentar, aún si proviene de mayorías parlamentarias (...) y vínculos porque los poderes de los estados están obligados a efectivizarlos" (Ávila, 2008, p. 22). En base a ello se percibe que no se puede hablar de democracia, si no existe en ella un efectivo mecanismo de división de poderes que permita hacer frente a los abusos de las autoridades estatales.

\section{I.2. La voluntad popular como cimiento del poder Legislativo}

Como se ha evidenciado en líneas anteriores, el fundamento del poder Legislativo es justamente trasmitir la voluntad de sus representados dentro de la Asamblea Nacional. Rousseau sostiene que "la voluntad es general o no lo es; es la del cuerpo del pueblo o solamente de una parte de él" (1981, p.52), por lo cual, para dicho autor aquella voluntad es indivisible y debe abarcar de manera efectiva lo deseado por todos los miembros del pueblo. Sin embargo, cabe recalcar que esto no debe ser entendido de manera literal, ya que de ser así el proceso de canalización de la voluntad popular se transformaría en utópico. 
Por ende, es menester comprender que para que una voluntad sea general, no siempre es necesario que sea unánime; pero lo crucial es que todas las voces que la componen sean tenidas en cuenta y que sus respectivos derechos sean igualmente protegidos y garantizados.

Solo en este sentido la ley podrá ser legítima, al perfilarse como un fiel reflejo de lo deseado por los gobernados. Asimismo, se constituiría en un mecanismo de igualdad que salvaguarde los intereses de todos los ciudadanos, sean estos o no afines al partido mayoritario.; pues las voluntades minoritarias estarían, de una u otra forma, plasmadas en la Asamblea por interpuesta persona de los representantes que los electores han elegido.

De este modo, no es posible afirmar la existencia de un sistema democrático, si en este no se toma en cuenta la voluntad popular, pues es en ella donde el poder encuentra su legitimidad. Por tanto, si eventualmente la voluntad popular dejara de verse plasmada en la Asamblea, y al contrario ésta se convirtiera en un órgano monopolizado, donde la posibilidad de las minorías políticas de manifestar sus ideas e intereses se viera relativizada, cabe cuestionarse ¿cuál sería la legitimidad y sustento del poder Legislativo?

\section{Análisis empírico de la atribución de esca- ños en la realidad nacional}

\section{II.1. Resultados de las elecciones legis- lativas ecuatorianas desde el retorno a la democracia.}

Una vez presentada la importancia de la representatividad como fundamento del poder Legislativo, resulta necesario estudiar la realidad nacional respecto a la atribución de escaños, para en base a ello analizar la legitimidad que adquiere el órgano Legislativo dentro del sistema democrático ecuatoriano. Así, a efectos de proporcionar al lector una perspectiva completa en cuanto a los resultados de las elecciones legislativas en el país; los mismos se encuentran plasmados de manera sistematizada a continuación, con el objetivo de apreciar claramente el porcentaje que los partidos mayoritarios han obtenido dentro del órgano Legislativo, siendo Alianza País, el primer movimiento político en obtener, desde el retorno a la democracia, una mayoría calificada en el Asamblea:

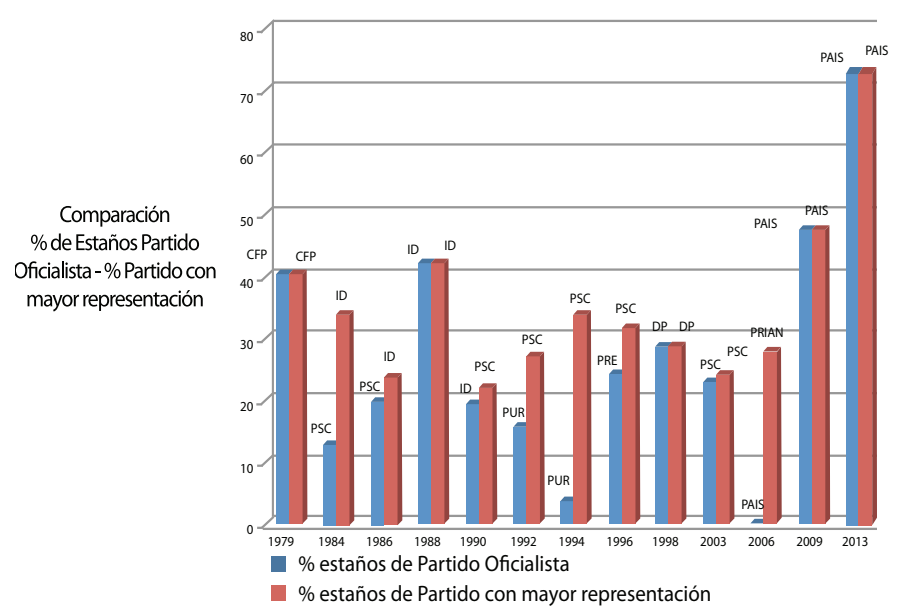

Figura 1. Fuente: Legislatin. Observatorio del poder Legislativo en América Latina.

\section{II.2. Aplicación del método} D'Hondt: ¿un sistema que favorece la monopolización del órgano Legislativo?

Tras la obtención de los desproporcionales resultados en las últimas elecciones legislativas, mucho se ha dicho respecto al método D'Hondt como una plausible causal de aquel corolario. Por ello, es indispensable remitirnos al referido método en el presente ensayo, a efectos de determinar si efectivamente es posible establecer un nexo entre los resultados obtenidos y el método empleado.

Si bien la comprensión matemática del sistema D'Hondt es crucial para un completo entendimiento del funcionamiento del mismo; ello es ajeno a los lineamientos del presente artículo. Por lo que, en esta parte, tan solo se explicará de manera general los principales preceptos de aquel sistema a efectos de generar en el lector un entendimiento razonable del mismo.

El método D'Hondt consiste, de manera general, en:

(...) sistemas de representación proporcional por lista basadas en el método de divisor o cifra mayor. Los votos de los distintos 
partidos o agrupaciones se dividen entre la serie consecutiva de números naturales 1,2 , 3 etcétera. Los escaños se le asignan a las organizaciones políticas de acuerdo con los resultados o cifras mayores que van generando las sucesivas operaciones. (Reynolds, A., Reilly, B., \& Ellis, A. , 2006, p. 196).

Así, se debe enfatizar respecto a que el sustento del referido sistema, recae en que los votos adquieren fuerza, y por tanto significancia, siempre y cuando el ciudadano haya votado por los demás candidatos de la lista, pues caso contario el voto individual se fragmentará y perderá eficacia. En base a ello se sostiene que la “[la fórmula del método] D'Hondt tiende a ser la más favorable para los partidos más grandes" (Reynolds, A., Reilly, B., \& Ellis, A. ,2006, p.196), lo cual implica un correlativo perjuicio en la aptitud de los partidos más pequeños para ser verdaderamente representados y considerados en la política legislativa nacional.

En este sentido, el Tribunal Constitucional declaró en 2004 la inconstitucionalidad del artículo 106 de la Ley de Elecciones- norma que estipulaba el uso del método de Hondt para la atribución de escaños en elecciones pluripersonales- al considerar que “el método D'Hont [sic] (...) resulta no ser el sistema adecuado para conciliar el espíritu que consagró constitucionalmente el principio de listas abiertas con el representación proporcional de las minorías" (Resolución del Tribunal Constitucional, Registro Oficial No.282:1 de marzo de 2004, p. 4). Asimismo, aquel criterio se vio respaldado a través del informe del Segundo Debate del Proyecto de Ley Orgánica Reformatoria a la Ley Orgánica Electoral, del cual se desprende que el método D'Hondt puede ser visto como perjudicial para las minorías, así:

(...) uno de los temas más polémicos y debatidos en el seno de la Asamblea fue la reforma del método de adjudicación de escaños. Mientras los proponentes de la reforma argumentaron que el sistema de divisores continuos unificaba el sistema de adjudicación de escaños establecido para elecciones pluripersonales (cfr. art. 164 del Código de la Democracia), fortalecía a las organiza- ciones políticas y aumentaba la gobernabilidad; los críticos de la reforma sostenían que el método D'Hondt no daba cabida a la representación de las minorías y favorecía a los partidos más fuertes (Informe para el segundo debate del Proyecto de Ley Orgánica Reformatoria a la Ley Orgánica Electoral y Organizaciones Políticas de la República del Ecuador: 2001, p. 9).

Ahora bien, pese a ello cabe cuestionarnos la implicación empírica que dicho sistema ha generado en nuestro país, para en base a ello determinar si es plausible atribuir un nexo directo entre el método empleado y la proporcionalidad o no de los resultados obtenidos en las últimas elecciones legislativas. Para ello es menester analizar de manera empírica las implicaciones de la aplicación del referido método a través de la comparación de los resultados de dos elecciones legislativas en las cuales dicho método fue empleado: en el 2002 y en el 2013.

En este sentido, se constata que en el 2002 el partido que más escaños obtuvo fue el Partido Social Cristiano (PSC) con 26 diputados de un total de 100 representantes Legislativos, lo que constituye el $26 \%$ (figura 1), mientas que en las últimas elecciones el partido mayoritario fue Alianza País con la obtención de 100 curules de un total de 137; lo que equivale al $73 \%$ (figura 1). De la comparación entre estos resultados se constata una amplia diferencia en la medida en la que en 2002 el partido con mayor número de representantes apenas alcanzaba un 26\% mientras que en la actualidad Alianza País ostenta per se una mayoría calificada en la Asamblea, lo que implica que el 27\% restante deberá dividirse entre las demás fuerzas políticas, lo cual relativiza la existencia de una real representación de las minorías, $\mathrm{y}$, por tanto, de todo el pueblo como tal.

Sin embargo, pese al amplio margen entre dichos resultados, en ambos se empleó el método D'Hondt. De esta manera, se percibe que no es posible atribuirle a este método en sí la obtención de resultados disparejos en la atribución de curules en la Asamblea, pues nuestra historia electoral no nos

1 Remitirse al cuadro expuesto en el título precedente del presente artículo. 
permite desprender un nexo de causalidad entre uno y otro factor. Por tanto, no se puede asegurar que el referido sistema genere imposibilidad de representación de las minorías políticas en el órgano Legislativo.

Efectivamente, pese a las críticas que la incorporación de dicho método en nuestro sistema electoral ha generado, esto no constituye una explicación efectiva a la desproporcional representación que se obtuvo tras las últimas elecciones legislativas. Por lo que este corolario se ve influenciado por distintos factores, tales como las tendencias políticas de las mismas, el apoyo popular, el discurso político entre otros. No obstante, más allá de los factores que conllevan a dichos resultados, resulta indispensable analizar la legitimidad de una mayoría calificada en manos de un solo partido-sin importar su ideología- dentro de la Asamblea Nacional.

\section{Conclusiones: ¿Existencia de una verdade- ra representación popular en la Asamblea Nacio- nal?}

Tras el estudio realizado anteriormente, resulta evidente presenciar la existencia de una verdadera complejidad en cuanto a la representación proporcional de la sociedad en el órgano Legislativo. Así, se constata que sin importar qué método se emplee, queda abierta la posibilidad de una ausencia de representación real de las minorías en la Asamblea Nacional. Al contrario, la causa de la desproporcional representación, que existe actualmente dentro de nuestro órgano Legislativo, halla razón en distintos factores tales como: el inmenso apoyo popular hacia el oficialismo; la ausencia de un voto reflexivo que implique por parte de los ciudadanos una responsabilidad previa con un respectivo análisis de los candidatos en sí más allá del partido o movimiento que representen; y el impacto que el discurso político presidencial ha logrado en los votantes, a través del uso de slogans que invitan a votar en plancha sin que se genere una verdadera consciencia respecto a las consecuencias que aquello implica dentro de un Estado Derecho. En este sentido, cabe cuestionarnos si ¿los resultados obtenidos irrumpen con la naturaleza del sistema democrático, en el cual, como se señaló con anterioridad, la voluntad del pueblo constituye la fuente de poder?
La relación entre el Ejecutivo y el Legislativo es sumamente delicada, pues de no existir en la Asamblea una mayoría afín al Ejecutivo, éste perdería la potestad de gobernar. En este sentido, se daría una pugna de poderes, frente a la cual, los partidos o movimientos de oposición verían en la Asamblea una plataforma política que les permitiría deslegitimar al Presidente; lo cual, constituiría una desnaturalización del órgano Legislativo y, por tanto, un perjuicio para el sistema democrático.

Del otro lado de la moneda se encuentra una situación que, pese a ser opuesta a la mencionada, configura de igual modo una amenaza a la democracia: aquella consiste en una excesiva e ilimitada intromisión del Ejecutivo dentro del Legislativo- cuadro que se vuelve posible a través de una mayoría calificada del régimen oficialista dentro de la Asamblea Nacional. Por ende, no se trata de llegar a uno u otro extremo, pues la esencia de la verdadera democracia radica en encontrar un equilibrio: es decir un cuadro en el que por un lado el órgano Legislativo se configure de diversas opiniones e interés políticos- todos con la posibilidad de expresar su voz y de ser efectivamente escuchados- mientras que por otro lado, el Ejecutivo ostente la facultad de tomar decisiones y aquellas sean asimismo fiscalizadas y controladas por una Asamblea independiente y autónoma.

Es precisamente por ello que indispensable recalcar el hecho de que en las últimas elecciones legislativas, se dio por primera vez en la historia del Ecuador- desde el retorno a la democracia- una mayoría calificada del partido oficialista en la Asamblea. Esto no puede, bajo ningún concepto pasar desapercibido, pues es nuestro deber como ciudadanos mantenernos atentos a esta trasformación en las relaciones entre el Ejecutivo y el Legislativo, pues esto pone evidentemente en riesgo uno de los principios rectores del Estado de Derecho: el principio de división de poderes.

Ante ello es fundamental manifestar que la Constitución de la República prevé que las decisiones más importantes dentro de la Asamblea sean tomadas mediante mayoría calificada (es decir que se requiere las dos terceras partes de votos a favor para tomar la decisión), pues se entiende que la obtención de aquel requisito garantizará que la medida haya sido aproba- 
da por un consenso de voluntades diversas que reflejan la existencia de un real acuerdo, pese a la diferencia partidaria, respecto al tema en cuestión.

No obstante, al existir actualmente una rotunda mayoría de Alianza País en la Asamblea- con el 73\% de los curules- aquellas decisiones, de mayor importancia y trascendencia, podrán ser tomadas únicamente con el consentimiento de los representantes de dicho partido; lo cual deja al margen la voluntad y opinión de los demás grupos políticos e implica una desnaturalización del órgano Legislativo, pues se abriría la posibilidad de tomar decisiones sin un verdadero diálogo y discusión entre los distintos intereses comprometidos.

Un claro ejemplo de esto se vive actualmente en el país en la medida en la que la Constitución establece en su artículo 441 que las enmiendas constitucionales podrán realizarse por dos vías: ya sea mediante consulta popular o por decisión de la Asamblea Nacional, siempre y cuando aquella se dé con la aprobación de las dos terceras partes de los asambleístas (es decir por mayoría calificada). La estipulación exclusiva de estas dos opciones se debe a que al ser la reforma de la Constitución una acción de suma relevancia e impacto en toda la población, esta debe realizarse por quien detiene el legítimo poder: el pueblo. Así, la norma considera que ya sea a través de un referéndum o a través de una mayoría parlamentaria calificada, ambas opciones son igualmente válidas porque a través de ellas se canaliza la voluntad popular.

No obstante, ante un poder Legislativo monopolizado en el que solo se exterioriza el querer del oficialismo, todo lo antes mencionado se ve relativizado, generando así un cuadro en el cual la presencia de una mayoría calificada en la Asamblea, pone en tela de duda la eficacia de aquel órgano. Así, el poder Legislativo corre el riesgo de convertirse en una mera manifestación de la voluntad del Ejecutivo frente a una Asamblea Nacional que no refleja la voluntad popular, sino tan solo la voluntad de los militantes del partido o movimiento político mayoritario. De ser este el caso, no solo se perdería la representatividad, sino que la Asamblea sería un ente disfuncional pues una de sus funciones primordiales - la capacidad de fiscalizar el actuar de los otros poderes del Estado- se vería asimismo obstruido por la injerencia del Ejecutivo.

Ante esto es fundamental mencionar la diferencia entre la democracia formal y la sustancial, sobre la cual enfatiza Ferrajoli; atendiendo la primera al procedimiento, es decir la necesidad de participación ciudadana; y la segunda refiriéndose al contenido, en la medida en la que no basta con que se respeten los procedimientos democráticos sino que se exige que una vez realizado aquello, las decisiones que emanen del poder político no atenten contra los derechos de las minorías (2011, p.16-17). El referido autor sostiene que "para que un sistema sea democrático, se exige al menos que la mayoría no tenga el poder de suprimir la posibilidad de que las minorías se conviertan en mayorías" (Ferrajoli, 2011, p.15); lo que quiere decir que las mayorías no deben opacar la opinión y las ideas de las minorías, porque al hacer aquello se extingue por completo la posibilidad de diálogo que es característica de todo sistema democrático; estando así ante una democracia netamente material y no sustancial.

Como se ha evidenciado en líneas anteriores, no es posible establecer un vínculo directo entre el método de asignación de escaños empleado y la representatividad de los resultados obtenidos, pues dicho corolario depende de diversos factores. No obstante, frente a esto, es el pueblo quien debe asumir un rol fiscalizador de la actividad legislativa. Así, es nuestro deber como ciudadanos mantenernos atentos a la presencia de esta inmensa mayoría en la Asamblea, pues solo podrá reflejar una real democracia en la medida en la que no implique abuso del poder. Resulta indispensable que exista y persista un respeto hacia las opiniones e intereses de las minorías. Si los grupos minoritarios no son efectivamente tomados en consideración antes de la aprobación de cualquier disposición legislativa; obtendremos únicamente leyes que no responden a la voluntad popular y que por tanto carecerán de legitimidad, quebrantando de este modo el sistema democrático.

La verdadera democracia exige mucho más que un mero procedimiento, ya que la soberanía popular no radica únicamente en las elecciones, sino 
que se manifiesta en el correcto ejercicio de los poderes estatales. Ello implica la necesidad de que la actividad que emane de cualquiera de los referidos poderes, responda a cabalidad al principio de división de poderes, a la autonomía y al respeto a las minorías; pues estas últimas pese a ser minorías, constituyen de igual modo el pueblo soberano.

\section{Referencias}

Asamblea Nacional de la República del Ecuador (2011). Informe para el segundo debate del Proyecto de Ley Orgánica Reformatoria a la Ley Orgánica Electoral y Organizaciones Políticas de la República del Ecuador, Código de la Democracia. Recuperado de: http://ppless. asambleanacional.gob.ec/alfresco/d/d/workspace/SpacesStore/f637ec82-d601-4238-bc2d-8d24d7fc0eb5/Informe\%20Primer\%20 Debate\%20Tr.\%2092166.pdf

Asamblea Nacional de la República del Ecuador (2009). Ley Electoral y de Organizaciones Políticas, Código de la Democracia. Registro Oficial: Suplemento 578 del 2009.

Ávila, R.(Ed.) (2008). La Constitución del 2008 en el contexto andino. Análisis desde la doctrina y el derecho comparado. Quito: Ministerio de Justicia y Derechos Humanos.

Díaz, E. (1975). Estado de Derecho y sociedad democrática. Madrid: Editorial Cuadernos para el Diálogo.

Ferrajoli, L. (2011). Principia Iuris: Teoría del derecho y de la democracia. Madrid: Editorial Trotta.

Ferrajoli, L. (2001). Pasado y futuro del estado de Derecho. Recuperado de: http://e-spacio. uned.es/fez/eserv.php?pid=bibliuned:filopoli-2001-17-0011\&dsID=pdf

Reynolds, A., Reilly, B., \& Ellis, A. (2006) Glosario en Diseño de sistemas electorales: El nuevo manual de IDEA internacional. Recuperado en: http://www.idea.int/publications/esd/ upload/anexo_b.pdf

Rousseau; J.J. (1981). Contrato social. Madrid: Espasa-Calpe.

Tribunal Constitucional (2004). Resolución del Tribunal Constitucional (2004). Registro Oficial No.282. Quito: Tribunal Constitucional. 